\title{
Visual laterality for letter comparison: Effects of stimulus factors, response factors, and metacontrol
}

\author{
JOSEPH B. HELLIGE and CHIKASHI MICHIMATA \\ University of Southern California, Los Angeles, California
}

\begin{abstract}
Right-handed subjects indicated whether two highly discriminable uppercase letters were the same or different. Letter pairs were projected to the left visual field/right hemisphere (LVF/RH) or the right visual field/left hemisphere (RVF/LH), or the same letter pair was presented to both visual fields simultaneously (bilateral trials). Laterality effects were not influenced by moderate blurring of the letters. However, on RVF/LH trials, reaction times were faster for same pairs than for different pairs. This effect was absent on LVF/RH trials, suggesting a qualitative difference in the mode of processing for the two unilateral trial types. The pattern of results on bilateral trials was identical to that obtained on RVF/LH trials. This suggests that on bilateral trials, the subjects employed the mode of processing characteristic of RVF/LH trials, perhaps indicating assertion of metacontrol by the left cerebral hemisphere.
\end{abstract}

Recent reviews have indicated that a variety of input and task factors can influence the pattern of visual laterality effects (e.g., Hellige \& Sergent, 1986; Sergent \& Hellige, 1986). The present experiment was designed to extend the investigation of some of these factors. During the experiment, observers indicated as quickly as possible whether two uppercase letters were identical or not. The letters used $(T, H$, and $X)$ were chosen because they are highly discriminable on the basis of their outer contours. The experiment's purposes follow.

\section{Stimulus Clarity}

In previous letter-matching studies involving letters that are more difficult to discriminate, it has been found that moderate blurring of the stimuli impairs performance more when the stimuli are projected to the right visual field/left hemisphere (RVF/LH) than when the stimuli are projected to the left visual field/right hemisphere (LVF/ RH) (e.g., Jonsson \& Hellige, 1986). The stimuli in these earlier studies were sufficiently difficult to discriminate so that moderate blurring did, in fact, increase errors and reaction time. One purpose of the present experiment was to determine whether a similar clarity (clear versus blurred) $\times$ visual field interaction would be obtained with stimuli that are so easy to discriminate that there would be no main effect of blurring.

This question is interesting for the following reason: One explanation of the clarity $\times$ visual field interaction obtained in earlier experiments has been that the right cerebral hemisphere is more resistant than the left cerebral hemisphere is to the effects of stimulus degradation,

This research was supported in part by Research Grant BNS-8608893 awarded by the National Science Foundation to Joseph B. Hellige. Correspondence may be addressed to Joseph B. Hellige, Department of Psychology, University of Southern California, Los Angeles, CA 90089-1061. perhaps because it is dominant for certain aspects of visuoperceptual analysis (e.g., Jonsson \& Hellige, 1986; Michimata \& Hellige, 1987; Sergent \& Hellige, 1986). According to this so-called stimulus-perceptibility hypothesis, clarity should only interact with visual field to the extent that the specific manipulation employed (in this case, blurring) actually interferes sufficiently with stimulus perceptibility to reduce the level of performance. Thus, the presence of a clarity $\times$ visual field interaction in the absence of a clarity main effect would present difficulty for the stimulus-perceptibility hypothesis.

An alternative explanation of previous clarity $\times$ visual field interactions has been given in terms of hemispheric differences in the utilization of information conveyed by higher versus lower channels of visual spatial frequency (e.g., Jonsson \& Hellige, 1986; Michimata \& Hellige, 1987; Sergent, 1985; Sergent \& Hellige, 1986). Specifically, it has been suggested that the left and right hemispheres are biased toward efficient use of higher and lower spatial frequencies, respectively. Accordingly, a manipulation like blurring (which selectively eliminates high spatial frequencies but leaves low frequencies intact) is predicted to shift visual field differences in the direction of an LVF/RH advantage. From this point of view, the same clarity $\times$ visual field interaction should be obtained, regardless of whether or not there is a main effect of clarity.

\section{Same/Different Stimulus Type and Metacontrol}

When subjects indicate whether two simultaneously presented letters are the same or different, it is often the case that reaction times are faster for same than for different pairs. This effect is referred to as the fast-same effect, and its interpretation is a matter of considerable speculation (e.g., Farell, 1985, 1988). When letter pairs are presented to either the LVF/RH or the RVF/LH on 
each trial, it is often the case that the fast-same effect is restricted to RVF/LH trials (e.g., see Bagnara, Boles, Simion, \& Umilta, 1983; Egeth \& Epstein, 1972), producing a same/different $\times$ visual field interaction. As Bagnara et al. argue, this result suggests that the left and right hemispheres perform the letter-comparison task in qualitatively different ways (see Bagnara et al., for consideration of what these different modes of processing might be).

The fact that the fast-same effect is restricted to RVF/ LH trials, together with the fact that the fast-same effect is typically found in studies that present the letter pairs in central vision (when the information is accessible to both hemispheres), suggests that the mode of letter comparison favored by the left hemisphere dominates this task when both hemispheres have access to the same stimulus information. In order to test this, the letter pairs in the present experiment were projected briefly to the LVF/RH or to the $\mathrm{RVF} / \mathrm{LH}$, or the same letter pair was projected simultaneously to both visual fields and hemispheres (the bilateral condition). That is, on bilateral trials, both hemispheres had simultaneous access to exactly the same stimulus information. To the extent that the pattern of same/different effects on bilateral trials matches that of one unilateral field but not the other, we have evidence about which hemisphere's preferred mode of processing dominates when both have equal access to relevant stimulus input (i.e., for the assertion of a type of "metacontrol" by one hemisphere).

Hellige, Jonsson, and Michimata (1988), who have used such bilateral trials in an experiment with drawings of faces, report that the qualitative pattern of results on bilateral trials was identical to the pattern of results on $\mathrm{RVF} / \mathrm{LH}$ trials, and that both were different from the pattern of results on $L V F / R H$ trials. On the basis of this, they argue that for most right-handers in their faceprocessing task, the left hemisphere asserts metacontrol under conditions in which both hemispheres receive the relevant stimulus information. The present experiment was designed to provide some indication of whether a similar finding might extend to a very different lettercomparison task.

\section{METHOD}

\section{Subjects}

Forty right-handed volunteers from introductory psychology classes (20 men, 20 women) with normal or corrected-to-normal visual acuity participated in the experiment. Ten subjects of each gender were assigned randomly to each of two groups, which were defined by the use of two response procedures (a two-button versus a four-button procedure).

\section{Apparatus}

The subject sat at a table facing a $44 \times 48 \mathrm{~cm}$ screen approximately $60 \mathrm{~cm}$ away. A black posterboard covered the screen, with two rectangular windows cut out so that stimuli could be presented to the left and right visual fields. There was a small circular opening midway between the two windows for presentation of a fixation dot. The subject's chin was placed on a padded rest with a forehead stabilization bar, in order to ensure that the subject's midline was perpendicular to the viewing screen. Centered on the table in front of the subject was a $17 \times 35.5 \mathrm{~cm}$ response console. On top of the console were two pairs of buttons, with the centermost button of each pair $7.5 \mathrm{~cm}$ from the center of the console. The two buttons within each pair were $7.5 \mathrm{~cm}$ apart. Two different response procedures were used for the two different groups of subjects. For the two-button procedure, only the outer buttons were used, and a card with the label same or different appeared over the left or right buttons (with side counterbalanced across subjects). For the fourbutton procedure, all four buttons were used. In this case, appearing above each of the two innermost buttons was a card with the same label (same or different, counterbalanced across subjects), and above each of the two outermost buttons was a card with the opposite label. Letter pairs and a fixation dot were rear-projected onto the viewing screen at the appropriate times, using a Gerbrands three-field tachistoscope (model G1176) equipped with two Kodak Carousel 850 slide projectors with Kodak Ektanar $f / 2.8$-in. lenses. Stimulus duration was controlled by a Gerbrands six-channel timer (model 300-6T). Summary statistics for each experimental session were computed with an Apple II microprocessor.

\section{Stimulus Materials}

The stimuli consisted of all possible pairs of the uppercase letters $T$, $H$, and X (Letraset Futura Medium). These three letters were chosen on the basis of pilot experiments indicating that they were so easy to discriminate that moderate blurring would be unlikely to produce a main effect. When projected on the viewing screen, the letters appeared as white on an opaque background, with one letter positioned approximately $0.8^{\circ}$ of visual angle above the other. The center of the projected letters was approximately $3.0^{\circ}$ of visual angle from the center of the screen, and each letter subtended approximately $0.8^{\circ}$ of visual angle horizontally and $1.0^{\circ}$ of visual angle vertically. The luminance of the letter pairs was approximately $4.0 \mathrm{~cd} / \mathrm{m}^{2}$. A fixation dot of similar luminance was projected at the appropriate times to the center of the screen, with a size of approximately $0.2^{\circ}$ of visual angle.

During the experiment proper, each subject received a total of 288 experimental trials. These trials were divided into blocks of 36 trials each. Within each 36-trial block, there were 6 trials of each type, defined by the orthogonal combination of stimulus type (same/different) and visual field (LVF/RH, RVF/LH, bilateral). Within each block, the trial types were arranged randomly, with the restriction that no one trial type occur more than three times in a row. On half of the 36-trial blocks, the letters were clearly focused on the viewing screen, and for the other half, the letters were moderately blurred by defocusing the slide projector so that a point of light in the clear condition appeared fuzzy and covered an additional $0.2^{\circ}$ of visual angle in the blurred condition (corresponding to the Blur 2 condition of Jonsson \& Hellige, 1986). The order of clear and blurred trial blocks was counterbalanced across subjects.

\section{Procedure}

At the beginning of the experimental session, all the subjects were told to keep the appropriate fingers of the left and right hands on the response keys, and to direct their gaze toward the fixation dot when it appeared. The subjects were told to maintain eye fixation until after they had made their response on each trial. The subjects were also told that on each trial of the experiment a pair of capital letters would appear on the screen in the left window, the right window, or both windows simultaneously. It was emphasized that on bilateral trials, the letter pair presented in the left window would always be identical to the letter pair presented in the right window. The subjects were told to indicate as quickly as possible on each trial whether or not the two letters of the pair were identical to each other, with the specific method of responding explained to the two-button and four-button groups. The subjects in the two-button groups were told to use the index finger of the right or left hand to press the key with the appropriate label above it. The subjects in the four-button groups were told to respond by pressing either both index fingers or both middle fingers, depending on which response was correct.

For all subjects, each trial began with the onset of the fixation dot for $1 \mathrm{sec}$, followed immediately by a letter pair for $150 \mathrm{msec}$. The in- 
tertrial interval was $5 \mathrm{sec}$. Prior to the experimental trials, each subject received 24 practice trials with clear stimuli.

\section{RESULTS AND DISCUSSION}

For each subject, the percentage of errors and median reaction time of correct responses were computed for each trial type. The percentage of errors was too low (3.1\%) to allow a meaningful analysis. The reaction times of correct responses were subjected to an ANOVA that included gender of subject and response procedure as betweensubjects variables, and clarity, stimulus type, and visual field (LVF, RVF, bilateral) as within-subjects variables. The only effect involving the between-subjects variables was a significant main effect of response procedure $[F(1,36)=4.47, p<.05]$, with reaction times being faster with the two-button procedure $(M=601 \mathrm{msec})$ than with the four-button procedure $(M=695 \mathrm{msec})$. Given that there were no interactions involving response procedure, and no effects whatsoever of gender, the remaining results are presented collapsed across these variables.

\section{Stimulus Clarity}

As anticipated, there was no main effect of clarity in the present experiment $[F(1,36)=2.18, p>.10$; clear $M=648 \mathrm{msec}$, blurred $M=652 \mathrm{msec}$. This is in contrast to the main effect of this level of blurring in more difficult stimulus-discrimination tasks (e.g., Jonsson \& Hellige, 1986; Michimata \& Hellige, 1987). Also in contrast to these more difficult tasks, there was no clarity $x$ visual field interaction in the present experiment $[F(2,72)<1.0]^{1}$

As noted in the introduction, the presence of a clarity $X$ visual field interaction in the absence of a clarity main effect would have presented difficulty for the stimulusperceptibility hypothesis and favored an explanation in terms of visual spatial frequency. As it stands, however, the stimulus-peceptibility hypothesis remains a plausible explanation of the effects obtained in earlier studies. It is interesting that the level of blurring used in the present experiment was equivalent to the level shown to produce a clarity $\times$ visual field interaction in earlier experiments that included stimuli more difficult to discriminate (Jonsson \& Hellige, 1986; Michimata \& Hellige, 1987). Accordingly, the present results indicate that removal of a particular range of high spatial frequencies per se does not guarantee a shift in the visual laterality pattern.

\section{Same/Different Stimulus Type and Metacontrol}

There was a significant main effect of stimulus type $[F(1,36)=12.47, p<.002$; same $M=635 \mathrm{msec}$, different $M=661 \mathrm{msec}$, and a significant stimulus type $\times$ visual field interaction $[F(2,72)=5.82, p<.005]$. This interaction is displayed in Figure 1 . With respect to the LVF/RH and RVF/LH trials, it can be seen that for $\mathrm{RVF} / \mathrm{LH}$ trials, reaction times are faster to same pairs than to different pairs $(p<.01)$, whereas there is no significant effect of stimulus type for LVF/RH trials. As a result, when analysis is restricted to the unilateral trials, the stimulus type $\times$ visual field interaction continues to be significant $[F(1,72)=4.24, p<.05]$. The fact that the fast-same effect was obtained on RVF/LH trials but not on LVF/RH trials is consistent with earlier findings (e.g., Bagnara et al., 1983; Egeth \& Epstein, 1972). Although the interpretation of this effect must remain somewhat speculative, it is consistent with a recent report that the left hemisphere is more sensitive than the right hemisphere is to the common features of two stimuli (Frost \& Gati, 1989). Regardless of the interpretation that is preferred, the fact that the fast-same effect was present on RVF/LH trials but not on LVF/RH trials indicates that comparison of the letters used in the present experiment is qualitatively different as a function of which hemisphers: received the stimuli. The presence of such a stimulus type $X$ visual field interaction is useful in examining the pattern of results on bilateral trials.

As Figure 1 shows, performance on bilateral trials was identical to performance on RVF/LH trials (both show the fast-same effect) and different from performance on LVF/RH trials. This is especially obvious when one considers the stimulus type $\times$ visual field interaction just discussed. These observations are corroborated by an ANOVA comparing RVF/LH with bilateral trials, in which no effects even approached statistical significance. In contrast, when the combined results of RVF/LH and bilateral trials were compared with LVF/RH trials, there was a significant stimulus type $\times$ visual field interaction $[F(1,36)=7.90, p<.01]$. These results did not depend

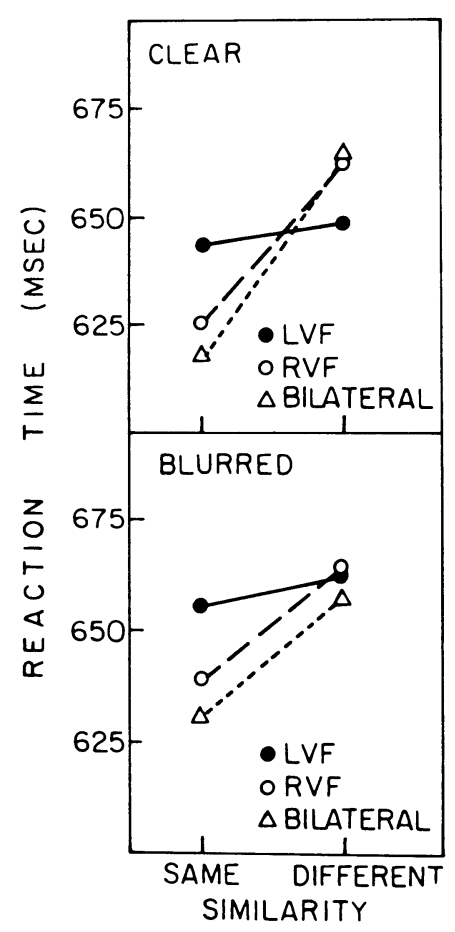

Figure 1. Reaction time to same and different letter pairs for stimuli presented to LVF, RVF, and bilateral locations. Results with clear and blurred stimuli are shown in the upper and lower panels, respectively. 
on which response procedure the subjects used, or on whether the stimuli were clear or blurred.

As noted in the introduction, Hellige, Jonsson, and Michimata (1988; see also Hellige, 1987) have used bilateral projection of the same information to both visual fields in a study involving the comparison of drawings of faces. Their results are similar to those of the present experiment, in the sense that the pattern of performance for bilateral trials was the same as the pattern for RVF/LH trials, and both were different from LVF/RH trials. One parsimonious explanation for these results is that when stimulus conditions allow right-handers to choose between two modes of processing, one preferred by the left hemisphere and the other by the right, they are biased toward the mode of processing that is characteristic of the left hemisphere. Further studies including bilateral as well as unilateral stimulus presentation are needed to determine both the extent to which such a bias is characteristic of right-handers, regardless of experimental task, and the manner in which such biases depend in systematic ways on specific task demands.

\section{REFERENCES}

Bagnara, S., Boles, D. B., Simion, F., \& Umilta, C. (1983). Symmetry and similarity effects in the comparison of visual patterns. Perception \& Psychophysics, 34, 578-584.

EgETH, H., \& EPSTEIN, J. (1972). Differential specialization of the cerebral hemispheres for the perception of sameness and difference. Perception \& Psychophysics, 12, 218-220.

FARELL, B. (1985). Same-different judgments: A review of current controversies in perceptual comparisons. Psychological Bulletin, 98 , 419-456.
FARELL, B. (1988). Comparison requirements and attention in identicalnonidentical stimulus discrimination. Journal of Experimental Psychology: Human Perception \& Performance, 14, 707-715.

Frost, R., \& GATI, I. (1989). Comparison of the geometric and contrast models of similarity by presentation of visual stimuli to the left and right visual fields. Brain \& Cognition, 9, 1-15.

HeLuIGE, J. B. (1987). Interhemispheric interaction: Models, paradigms and recent findings. In D. Ottoson (Ed.), Duality and unity of the brain (pp. 454-465). Hampshire, England: MacMillan Press.

Hellige, J. B., Jonsson, J. E., \& Michimata, C. (1988). Processing from LVF, RVF and BILATERAL presentations: Metacontrol and interhemispheric interaction. Brain \& Cognition, 7, 39-53.

Hellige, J. B., \& SERGENT, J. (1986). Role of task factors in visual field asymmetries. Brain \& Cognition, 5, 200-222.

Jonsson, J. E., \& Hellige, J. B. (1986). Lateralized effects of blurring: A test of the visual spatial frequency model of cerebral hemisphere asymmetry. Neuropsychologia, 24, 351-362.

Michimata, C., \& Hellige, J. B. (1987). Effects of blurring and stimulus size on the lateralized processing of nonverbal stimuli. Neuropsychologia, 25, 397-407.

SERGENT, J. (1985). Influence of task and input factors on hemispheric involvement in face processing. Journal of Experimental Psychology: Human Perception \& Performance, 11, 846-861.

Sergent, J., \& Hellige, J. B. (1986). Role of input factors in visual field asymmetries. Brain \& Cognition, 5, 174-199.

\section{NOTE}

1. An additional group of $\mathbf{4 0}$ subjects ( 20 men, 20 women) performed the same letter-classification task as did subjects in the present experiment, but without any bilateral trials included. The results obtained from this group were identical to the results obtained on the unilateral trials of the present experiment. That is, the inclusion of bilateral trials has no influence on the pattern of effects obtained on unilateral trials.

(Manuscript received February 7, 1989.) 